\title{
Does Pattern Recognition on OCT Angiography (OCTA) Help in the Prognosis of Neovascular AMD (nAMD)?
}

\section{Manoj Soman, Sameer I*, Asmita Indurkar, Ravi RV, Narendra Meel and Unnikrishnan Nair}

Department of Vitreo-Retinal Service, Chaithanya Eye Hospital and Research

Institute, Thiruvananthapuram, Kerala, India

*Corresponding Author: Sameer I, Department of Vitreo-Retinal Service, Chaithanya Eye Hospital and Research Institute, Thiruvananthapuram, Kerala, India.
Received: November 05, 2021

Published: November 18, 2021

(C) All rights are reserved by Sameer I., et al.

\section{Abstract}

Aim: To study the morphological patterns of nAMD on OCTA, compare with ICGA the gold standard and to ascertain whether pattern identification can help in prognosis of the disease and follow up.

Materials and Methods: Retrospective study of 103 nAMD patients who visited retina clinic at Chaithanya eye hospital, Trivandrum. Patients who underwent comprehensive fundus evaluation including FFA/ICGA, SD-OCT and OCTA on Heidelberg platform with minimal follow up of 1 year were analyzed. Vascular networks patterns identified on OCTA were analyzed. Area of the lesion on OCTA was correlated with FFA/ICGA at baseline and during treatment. Patients underwent 3 loading anti VEGF injections, followed by PRN on basis of OCT/ICGA findings and the data correlated with OCTA findings. On OCTA, good anatomical outcome was defined as decreased membrane size and decreased/absent SRF during follow up.

Results: Network patterns were more discernable on OCTA than ICGA - Sea fan (42\%), filamentous (20\%), Fish net (17\%), ill-defined $(13 \%)$ and lacy wheel (8\%). Out of 8 eyes with ill-defined membranes on ICGA, OCTA revealed fish-net pattern in 2 eyes while 6 remained ill-defined. Size of lesion was comparable between OCTA and ICGA (Mean horizontal and vertical diameters 2621 Vs 2445 and 2535 Vs $2189 \mu \mathrm{m}$ respectively). Post treatment size of network decreased on OCTA. Good anatomical outcomes were seen in $100 \%$ Lacy wheel eyes, $84.6 \%$ of sea-fan, $50 \%$ of filamentous and $50 \%$ of ill-defined pattern eyes. Fish net pattern was resistant to anti-VEGF therapy.

Conclusion: OCTA could classify nAMD lesions morphologically into various patterns more clearly and elaborately. Certain morphologies on OCTA had better anatomical outcomes and identification of these at baseline could help in prognostication and patient counseling. OCTA Fish net pattern not described in literature were found to be resistant to Anti-VEGF therapy.

Keywords: Fundus Fluorescein Angiography (FFA); OCT Angiography (OCTA); Indocyanin Green Angiography (ICGA)

\section{Introduction}

Age-related macular degeneration (AMD) is one of the most common cause of visual impairment in older population [1]. Fundus Fluorescein Angiography (FFA) along with Indocyanin Green Angiography (ICGA) is considered to be the gold standard investigation for neovascular AMD (nAMD). Being invasive procedure and not depth resolved, it has got its own disadvantages.
With recent advent of Optical Coherence Tomography Angiography (OCTA), non-invasive, safe, and rapid image acquisition has become possible, creating detailed 3D-angiograms of retinal and choroidal vasculature [2,3]. OCTA provides vascular imaging via motion contrast processing of decorrelation signals. However, there are limitations with the image quality due to projection and motion artifacts, interfering accurate visualization of Choroidal Neovascular Membrane (CNVM) [4]. 
Several studies investigated the use of OCTA in describing the anatomical pattern of CNVM in nAMD patients. They have described them variously as well or poorly circumscribed, "sea-fan" or "medusa" and "tangled" or "filamentous" [5-7]. The correlation of OCTA findings and CNV activity has also been investigated. Coscas., et al. [8]. Suggested 5 criteria to evaluate an active CNV including shape, branching, anastomoses, vessels termini and peri-lesional hypo-intense halo. However, clinical implications of different morphological patterns of CNV are still unknown. Also the relationship between these OCTA parameters and disease prognosis after antiVEGF treatment remains inconclusive.

Therefore, we designed this study to evaluate OCTA patterns in late AMD and compared to the current gold standard, FFA/ICGA and studied if these could help in predicting anti-VEGF responders and ascertain whether pattern identification can help in prognosis of the disease and follow up.

\section{Materials and Methods}

This retrospective study reviewed 103 ARMD patients with Choroidal Neovascularization who visited the retina clinic at a tertiary eye hospital between November 2017 and May 2019. Patients with a minimum follow up of 1 year were included in the study. All the patients underwent a comprehensive eye examination including slit-lamp biomicroscopy, tonometry, fundus examination with indirect ophthalmoscopy, fundus fluorescein angiography (FFA), indocyanine green angiography (ICGA), spectral-domain OCT (SDOCT), and OCT angiography (OCTA) on Hiedelberg Spectralis platform whenever indicated. Patients were excluded if they had concomitant ophthalmology conditions such as media opacities which limited the ability to take good images or if they had other causes of suspected CNV or could not follow up for 1 year.

Diagnosis of CNVM was made with OCT by assessing sub retinal or intra-retinal fluid, PED and reflectivity above or below RPE. FFA was done to characterize occult, classic and mixed CNVMs and ICG angiography was done to identify the network and to describe its pattern. Membranes on ICGA were classified as "sea-fan", "lacy wheel" and "ill-defined" patterns. Maximum vertical and horizontal height of lesions were measured.

OCTA was performed in all cases and analyzed for presence of a vascular network either in the avascular slab or choriocapillary slab and a composite slab was used for describing the morphol- ogy. Membranes on OCTA were classified as "sea-fan", "lacy wheel" "filamentous" and "ill defined" patterns. If a pattern which does not fit into the above pattern was seen, it was given a description. The morphology, content and area were also assessed. Segmentation and RPE contours were checked for accuracy in every patient. Manual segmentation of OCTA was done if required. Appearance of CNV on OCTA images and corresponding OCT B-scans were assessed and evidence of activity like presence of SRF, IRF or sub-RPE fluid was recorded. Area of the lesion measured on OCTA was correlated with that obtained on ICGA.

Patients diagnosed to have active disease were treated with 3 loading doses of intravitreal anti-VEGF injections followed by PRN on basis of OCT/ICGA findings. After 3 injections, patients were reassessed for the presence of activity on the basis of structural OCT primarily and ICGA was done whenever required. At each visit at monthly intervals, OCT/OCTA was done and data on membrane size, morphology, presence of fluid and modification of PED on OCT were recorded. Retreatment was based on standard guidelines using clinical, OCT and angiography criteria. Treatment modifications were not made on OCTA changes alone. However in some instances where ICGA was not repeated, OCTA findings in correlation with structural OCT was used to help treatment decisions. However for purpose of analysis, good anatomical outcome was defined on OCTA as decreased membrane size and decreased or absent SRF on structural OCT.

\section{Observation and Results}

103 patients with nAMD were included in our study. Mean age of our study group was 67.64 . In our study $54.9 \%$ were females while $45.1 \%$ were males. Common associated systemic risk factors were hypertension (43\%), diabetes (31.4\%) and ischemic heart disease (15.7\%). FFA revealed classic CNVM in 29\% eyes, occult CNVM in $60.5 \%$ eyes and rest had mixed pattern $(10.5 \%)$. When FFA findings were compared with OCTA findings, 25 out of 31 eyes $(81.8 \%)$ with classic CNVM revealed lacy wheel pattern, 3 eyes a sea fan (9\%) and rest 3 eyes revealed fish net pattern (9\%) on OCTA. Fish net was a new pattern described on OCTA which was well defined membrane with no central stalk like lacy wheel or a leading stalk like sea fan. These were well defined with a net like appearance unlike the relatively poorly defined tangled network or the ill defined membranes. Out of 62 eyes with occult CNVM on FFA, 24 eyes revealed sea-fan pattern (39\%), 16 eyes filamentous 
(26\%), 6 eyes (8.6\%) lacy wheel and fish net pattern each and 11 eyes had ill-defined membrane (17.3\%). Thus 51 out of 62 eyes had a describable pattern of membrane on OCTA (82.6\%). 10 eyes with mixed CNVM on FFA revealed a filamentous pattern on OCTA in 7 eyes and sea fan pattern in 3 eyes.

\begin{tabular}{|l|c|c|c|c|c|}
\hline \multirow{2}{*}{ FFA } & \multicolumn{5}{|c|}{ ICGA } \\
\cline { 2 - 6 } & $\begin{array}{c}\text { Lacy } \\
\text { Wheel }\end{array}$ & Seafan & Fishnet & Filamentous & Ill-defined \\
\hline CLASSIC & $23(73 \%)$ & 0 & 0 & 0 & $8(27 \%)$ \\
\hline OCCULT & $5(8.6 \%)$ & $27(43.4 \%)$ & 0 & $19(30.4 \%)$ & $11(17.3 \%)$ \\
\hline MIXED & 0 & 3 & 0 & $5(50 \%)$ & 2 \\
\hline
\end{tabular}

Table 1: Comparison of FFA vs ICGA patterns.

Commonest patterns were sea-fan (43.4\%), filamentous (30.4\%), ill-defined (17.3\%) and lacy wheel (8.6\%) pattern in order of frequency.

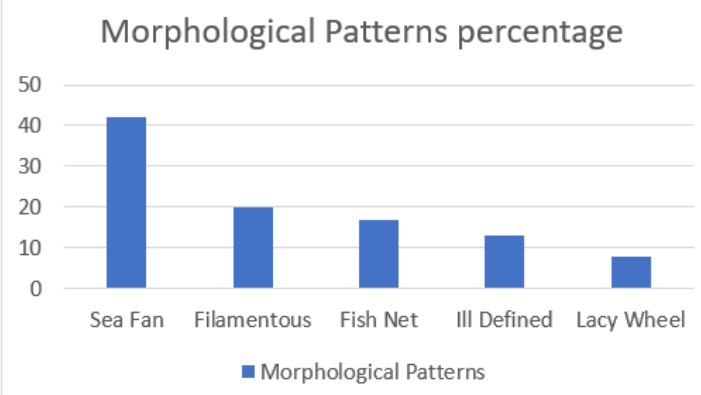

Figure 1: Prevalence of morphological patterns in study eyes.

\begin{tabular}{|l|c|c|c|c|c|}
\hline \multirow{2}{*}{ ICGA } & \multicolumn{4}{|c|}{ OCTA } \\
\cline { 2 - 6 } & $\begin{array}{c}\text { Lacy } \\
\text { wheel }\end{array}$ & $\begin{array}{c}\text { Sea } \\
\text { fan }\end{array}$ & Filamentous & Ill defined & Fishnet \\
\hline Lacy wheel & 28 & & & & \\
\hline Sea fan & & 30 & & & \\
\hline Filamentous & & & 24 & & 3 \\
\hline ILL Defined & & & & 19 & 5 \\
\hline
\end{tabular}

Table 2: Comparison of ICGA vs OCTA patterns.

Classic CNVM eyes had a Lacy wheel pattern in 73\% eyes and illdefined pattern in 27\% eyes in ICGA. Occult CNVM demonstrated a membrane in all cases on ICGA. Out of the 19 ill-defined membranes on ICGA, 5 eyes had a well-defined fish netpattern (25\%) while the rest 14 eyes (75\%) remained ill-defined on OCTA as well.
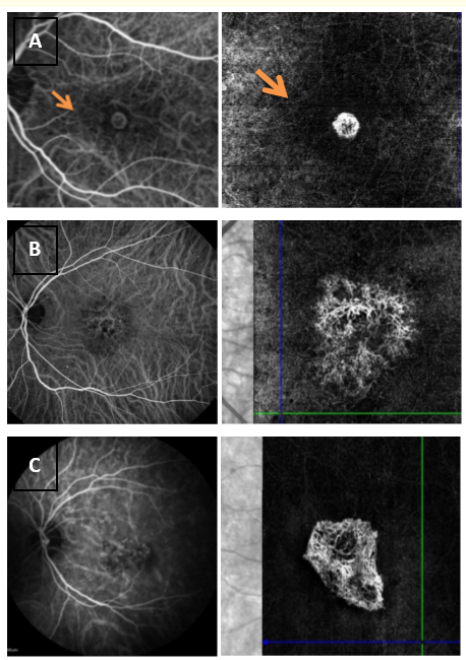

Figure 2: A; Showing lacy wheel pattern on ICGA as well as on OCTA. B; Sea fan on ICGA and OCTA, C; Ill-defined pattern on ICGA and OCTA.

\begin{tabular}{|l|c|c|c|c|}
\hline & n & Mean & SD & p-value \\
\hline SIZE H_OCTA & 39 & 2621.25 & 865.06 & \multirow{2}{*}{0.362} \\
\cline { 1 - 4 } SIZE H_ICG & 39 & 2445.92 & 1184.27 & \\
\cline { 1 - 4 } SIZE V_OCTA & 39 & 2535.17 & 984.22 & \multirow{2}{*}{0.163} \\
\cline { 1 - 4 } SIZE V_ICG & 39 & 2189.42 & 1044.92 & \\
\hline
\end{tabular}

Table 3: Comparison of size of the lesion in OCTA and ICGA.

(H- Maximum Horizontal Diameter, V- Maximum Vertical Diameter). The size of the lesion assessed by maximum horizontal and vertical linear diameter was comparable between OCTA and ICGA.

\begin{tabular}{|l|c|c|c|c|}
\hline SIZE H & $\mathbf{n}$ & Mean & SD & p-value \\
\hline PRE & 75 & 2295.5 & 876.5 & 0.007 \\
\hline POST & 75 & 2098.9 & 956.7 & \\
\hline SIZE V & $\mathrm{n}$ & Mean & SD & P value \\
\hline PRE & 75 & 2329.4 & 959.3 & 0.013 \\
\hline POST & 75 & 2023.1 & 1088.5 & \\
\hline
\end{tabular}

Table 4: Comparison of pre injection and post injection size $(\mathrm{H})$ and (V) of the lesion. 


\begin{tabular}{|c|c|c|c|c|c|}
\hline \multirow[t]{2}{*}{ OCTA } & \multicolumn{2}{|c|}{ AREA PREINJECTION } & \multicolumn{2}{|c|}{ AREA POST INJECTION } & \\
\hline & $\mathrm{H}$ & $\mathrm{V}$ & $\mathrm{H}$ & $\mathrm{V}$ & \\
\hline MEDUSA HEAD & 1711 & 1306.5 & 1346 & 1005.5 & $\eta$ \\
\hline SEAFAN & 2296.6 & 2357.6 & 2094.1 & 1589 & 7 \\
\hline $\begin{array}{l}\text { FILAMENTOUS } \\
\text { /TANGLED }\end{array}$ & 1539 & 2035 & 1244 & 1800 & $\downarrow$ \\
\hline FISHNET & 2783.3 & 1973.6 & 2508 & 2062 & $\mathrm{v} \uparrow$ \\
\hline ILLDEFINED & 2460.3 & 2570.6 & 2297.8 & 2420 & I \\
\hline
\end{tabular}

Figure 3: Comparison of size in pre injection and post injection in different morphological patterns in OCTA.

Size of the lesion decreased in all lesions during follow up with anti-VEGF therapy except the greatest linear vertical diameter in fishnet pattern. SRF decreased in $42 \%$ eyes and fully resolved in 47.3\% eyes with AMD. SRF decrease was seen in all membranes except fish net and ill-defined networks wherein $50 \%$ of the lesions had persistent fluid. All the lacy wheel pattern and $84.6 \%$ of sea fan pattern demonstrated good anatomical outcomes. While only $50 \%$ of the filamentous and ill-defined membranes had a good outcome.

\section{Discussion}

OCTA has revolutionized the study of nAMD. OCTA of patients with exudative AMD show structural details of CNV lesions with great depth resolution and viewing them on cross section identifies their exact position, enabling classification as type I, type II, or type III CNV [9-11]. Sarraf and colleagues [6] described morphologic characteristics of type I CNV lesions on OCTA. In their study of 33 eyes with AMD they described 2 distinct appearances of type I membranes: a "medusa" form (55\% lesions) with vessels radiating in all directions from a large mainfeeder vessel and a "sea-fan" form $(25 \%)$ where most of the smaller vessels radiated from one side of a large feeder vessel. The remaining vascular membranes lacked distinct smaller vessels. Souied., et al. [12,13] have described OCTA features of type II and type III CNVMs in AMD. They described 2 morphologic patterns of type II CNV on SD-OCTA a "medusa-shaped" lesion and a "glomerulus-shaped" lesion. Eyes with type III CNV were characterized by a tuft-shaped high-flow vascular network arising from the deep capillary plexus in outerretinal layer with adjoining telangiectatic vessels. Thus all these OCTA based studies have shown that OCTA better defines the abnormal network in all types of CNVM.
In the past few decades, FFA and ICGA have been the investigative modality in diagnosing and evaluating CNVM. In our study among 103 nAMD eyes, FFA revealed classic CNVM in 29\% eyes, occult CNVM in $60.5 \%$ eyes while the rest had mixed pattern $(10.5 \%)$. Classic CNVM eyes had a Lacy wheel pattern in 73\% eyes and illdefined pattern in 27\% eyes in ICGA. In Occult CNVM, it is reported that ICGA may be a better modality because it reveals a defined membrane which is not obvious on FFA. In our study also occult CNVM eyes demonstrated a membrane in all cases on ICGA. The commonest pattern were sea-fan $(43.4 \%)$, filamentous $(30.4 \%)$, ill-defined $(17.3 \%)$ and lacy wheel $(8.6 \%)$ pattern in order of frequency.

In our study, when we compared the ICG findings in occult CNVM with that of OCTA, we found that all the 28 lacy wheel patterns and 30 sea fan patterns on ICGA also revealed similar identical patterns on OCTA. Out of the 27 poorly defined filamentous pattern on ICGA, 24 eyes (89\%) revealed filamentous pattern on OCTA and 3 eye $(11 \%)$ showed well defined fish-net pattern. Out of the 24 ill-defined membranes on ICGA, 5 eyes had a well-defined fish net pattern (25\%) and rest 19 eyes $(75 \%)$ remained ill-defined on OCTA as well. Our study thus demonstrates not only comparability of OCTA over ICGA but also its superiority in some forms like the illdefined and filamentous pattern where a definite defined network was seen on OCTA.

The new patterns that we have identified include the fish net pattern which consisted of eyes which did not have the typical lacy wheel pattern with characteristic eye and spoke appearance or the typical sea fan appearance with a feeder and network at one end. These networks however were as well defined as the above two networks and fell in between these two types. This like the above two networks probably represent more evolved membranes as they were seen in type $2 \mathrm{CNVM}$ eyes with characteristic classic FFA fluorescence. In support of this fact was the observation that filamentous or tangled network seen in ICGA of occult CNVM eyes may represent a poorly organized network as this was not seen in any eye with classic CNVM. It appeared that lacy wheel pattern best represented classic/type 2 CNVM. When the pattern is a non-lacy wheel pattern the likelihood of this lesion being type 1 or occult CNVM is high. Majority of these eyes had a sea fan or filamentous appearance. 
Several studies have thus demonstrated that OCTA can $[6,8,14,15]$ offer an unrivaled morphological characterization of CNV net that far exceeds FA, ICGA and structural OCT. Since OCTA image is not obscured by dye leakage, a clear depiction of CNV net is provided. Coscas., et al. [8] had compared the OCTA with traditional multimodal imaging in patients with exudative AMD and found that there was high inter-observer agreement both for treatment decision in conventional multimodal and for Patterns (I or II) defining on OCTA imaging analysis.

In our study the ICG membrane detection rate was 79\% for welldefined descriptive membranes and on OCTA was $84 \%$. When we compared the size of network on OCTA and ICGA we found that it was comparable. Thus not only that OCTA could detect almost all the membranes that were identified on ICGA but it was also reliable in assessing the size of the lesion accurately. This has implications during follow up when the decrease in size can be used as a marker for adequate treatment response and to define non-responders.

In our study however we found an association of a particular morphology on OCTA in eyes with AMD with good anatomical outcome. All the lacy wheel pattern eyes and $84.6 \%$ of sea-fan pattern eyes demonstrated good anatomical outcomes. While only $50 \%$ of the filamentous and ill-defined membranes had a good outcome.

Sarraf and colleagues [6] quantified lesions by measuring area, width of the largest caliber vessel, and density of vessels in Type 1 CNVM. Notably, there was also no significant change in the area and vessel density of type I CNV lesions at baseline and at follow-up imaging after further anti-VEGF therapy, although qualitatively there appeared to be attenuation of some of the finer vessels in some of the eyes. Thus authors hypothesize that the lack of change in morphology after anti-VEGF injections likely reflects the chronicity of the lesions.

However Sarraf and colleagues [16] had used SS-OCTA to quantify the responseof type II CNV lesions to anti-VEGF treatments in a patient with exudative AMD and showed that the lesion area decreased by $40 \%$ at 4 weeks after the $1^{\text {st }}$ injection and that the vascular density decreased by $50 \%$. At 4 weeks after $2^{\text {nd }}$ injection, the lesion size had further decreased by $30 \%$ and the vessel density by $25 \%$. They noted that the caliber of the main feeder vessel did not change and hypothesized that the larger feeder vessel may be more resistant to anti-VEGF therapy possibly due to the presence of over- lying pericytes that do not surround the smaller vessel branches of the membrane. Sarraf and colleagues [17] have also studied type III CNVMs with OCTA and noted that follow-up imaging after receiving an intravitreal aflibercept injection, the CNV lesion was no longer evident.

Similar to the above observations our study also revealed that the dimensions of the membrane on OCTA decreased with antiVEGF therapy during the last follow up when compared to baseline. This decrease in dimensions of the membrane was demonstrable in lacy wheel, sea fan, filamentous and ill-defined networks but not evident on fish net network. SRF decrease was seen in all membranes except fish net and ill-defined networks wherein 50\% of the lesions had persistent fluid. It thus appears that the newly described fish net appearance is associated with persistent fluid and no decrease in dimensions of the membrane in at least half the cases after anti VEGF treatment. Pattern based treatment protocols could thus evolve as also discussed by Marques JP., et al. [18].

In our study even though SRF decreased in $42 \%$ eyes and fully resolved in $47.3 \%$ eyes with AMD, OCTA revealed persistence of network in $100 \%$ eyes following anti-VEGF therapy even though the size of the lesion decreased. Coscas., et al. had reported that all eyes with SRF, IRF, or sub-RPE fluid on OCT B-scan were associated with the active CNV (requiring treatment), but not everyone without fluid accumulation was definitively as quiescent CNV (not requiring treatment). Thus absence of fluid may not always mean that the disease is quiescent and this could explain the idea why treat and extend protocol could be a better way to treat AMD. Also this could indicate when one needs to stop therapy especially in the setting of network that has disappeared on OCTA.

Thus OCTA evaluation and monitoring of eyes with nAMD is promising not only because of the non-invasive nature of the technique which allows comprehensive lesion monitoring and consistent repeatability, it also may allow for the advent of tailored and customized treatment regimens for wet AMD built upon the CNV characteristics at baseline and its response to treatment as discussed by Marques JP., et al. [18].

\section{Conclusion}

We could demonstrate that OCTA was comparable to ICGA, the current gold standard in the evaluation of Neovascular AMD. We also found that the size of network on OCTA and ICGA were com- 
parable. OCTA could also classify the lesions morphologically into various patterns much more clearly and more elaborately than ICGA and could identify new patterns. We also found that certain morphologies on OCTA have good anatomical outcomes and identification of these patterns at baseline could help in prognostication and patient motivation. We could identify a new pattern; the fishnet pattern which is a well-defined network with multiple branching and anastomos is not hitherto described in literature and found that this pattern was resistant to Anti-VEGF therapy.

\section{Bibliography}

1. Lim LS., et al. “ARMD”. Lancet 379 (2012): 1728-1738.

2. Jia Y., et al. "Quantitative OCTA of vascular abnormalities in the living human eye". Proceedings of the National Academy of Sciences of the United States of America 112.18 (2015): E2395E2402.

3. Spaide RF and Klancnik JM. "Retinal vascular layers imaged by FA and OCTA". JAMA Ophthalmology 133.1 (2015): 45-50.

4. Gass JD. "Biomicroscopic and histopathologic considerations regarding the feasibility of surgical excision of subfovealneovascular membranes". American Journal of Ophthalmology 18.3 (1994): 285-298.

5. Inoue $\mathrm{M}$ and Sarraf D. "A Comparison Between OCTA and FA for the Imaging of Type 1 neovascularization". Investigative Ophthalmology and Visual Science 57 (2016): 0CT314-323.

6. Kuehlewein L and Bansal M. "OCTA of Type 1 Neovascularization in ARMD". American Journal of Ophthalmology 160 (2015): 739-748.

7. Liang MC and de Carlo TE. "Correlation of SD-OCTA and Clinical Activity in nARMD”. Retina 36 (2016): 2265-2273.

8. Coscas GJ and Lupidi M. "OCTA Vs Traditional multimodal imaging in assessing the activity of exudative ARMD: A New Diagnostic Challenge". Retina 35 (2015): 2219-2228.

9. Boltz A., et al. "Choroidal blood flow and progression of ARMD in the fellow eye in patients with unilateral CNV". Investigative Ophthalmology and Visual Science 51 (2010): 4220-4225.

10. Bhutto I and Lutty G. "Understanding AMD: relationships between the photoreceptor/RPE/Bruch's membrane/choriocapillaris complex". Molecular Aspects of Medicine 33 (2012): 295-317.
11. Bhutto IA and Uno K. "Reduction of endogenous angiogenesis inhibitors in Bruch's membrane of the submacularregionineyes with ARMD". Archives of Ophthalmology 126 (2008): 670678.

12. El Ameen A., et al. "Type 2 neovascularization secondary to ARMD imaged by OCTA". Retina 35 (2015): 2212-2218.

13. Miere A and QuerquesG. "OCTA in early type 3 neovascularization”. Retina 35 (2015): 2236-2241.

14. Moult E., et al. "Ultrahigh-speeds wept-source OCTA in exudative AMD". Ophthalmic Surgery, Lasers and Imagingretina 45.6 (2014): 496-505.

15. Jia Y., et al. "Quantitative OCTA of choroidal neovascularization in ARMD”. Ophthalmology 121.7 (2014): 1435-1444.

16. Kameda T., et al. "Polypoidal choroidal vasculopathy examined with en face OCT". Clinical and Experimental Ophthalmology 35 (2007): 596-601.

17. Kuehlewein L., et al. "OCTA and sequential quantitative analysis of type 2 neovascularization after ranibizumab therapy". Eye (London) 29 (2015): 932-935.

18. Marques JP and Silva R. “OCTA in wet AMD”. Eye Science (2016).

\section{Volume 4 Issue 12 December 2021 (C) All rights are reserved by Sameer I., et al.}

